\title{
Magnetic analog of the isotope effect in cuprates
}

\author{
Rinat Ofer, ${ }^{1}$ Galina Bazalitsky, ${ }^{1}$ Amit Kanigel,,${ }^{1}$ Amit Keren, ${ }^{1}$ Assa Auerbach, ${ }^{1}$ James S. Lord, ${ }^{2}$ and Alex Amato ${ }^{3}$ \\ ${ }^{1}$ Physics Department, Technion-Israel Institute of Technology, Haifa 32000, Israel \\ ${ }^{2}$ Rutherford Appleton Laboratory, Chilton Didcot, Oxfordshire OX11 OQX, United Kingdom \\ ${ }^{3}$ Paul Scherrer Institute, CH 5232 Villigen PSI, Switzerland \\ (Received 30 October 2006; published 13 December 2006)
}

\begin{abstract}
We present extensive magnetic measurements of the $\left(\mathrm{Ca}_{x} \mathrm{La}_{1-x}\right)\left(\mathrm{Ba}_{1.75-x} \mathrm{La}_{0.25+x}\right) \mathrm{Cu}_{3} \mathrm{O}_{y}$ system with its four different families $(x)$ having a $T_{c}^{\max }(x)$ variation of $28 \%$ and minimal structural changes. For each family, we measured the Néel temperature, the anisotropies of the magnetic interactions, and the spin-glass temperature. Our results exhibit a universal relation $T_{c}=c J n_{s}$ for all families, where $c \sim 1, J$ is the in-plane Heisenberg exchange, and $n_{s}$ is the superconducting carrier density. This relates cuprate superconductivity to magnetism in the same sense that phonon-mediated superconductivity is related to atomic mass.
\end{abstract}

DOI: 10.1103/PhysRevB.74.220508

PACS number(s): 74.40.+k, 05.70.Ln, 74.25.Fy, 74.62.-c

The critical temperature for superconductivity $T_{c}$ in metallic superconductors varies with isotope substitution. ${ }^{1}$ This observation, known as the isotope effect, played a key role in exposing their mechanism for superconductivity. In contrast, the mechanism for superconductivity in the cuprate is still elusive, but is believed to be of magnetic origin. ${ }^{2}$ Verifying this belief would require an experiment similar to the isotope effect, namely, a measurement of $T_{c}$ versus the magnetic interaction strength $J$, with no other structural changes in the compounds under investigation. Here we present such an experiment using the $\left(\mathrm{Ca}_{x} \mathrm{La}_{1-x}\right)\left(\mathrm{Ba}_{1.75-x} \mathrm{La}_{0.25+x}\right) \mathrm{Cu}_{3} \mathrm{O}_{y}$ (CLBLCO) system with its four different superconducting families, for which maximum $T_{c}\left(T_{c}^{\max }\right)$ varies by $28 \%$. This is a large change compared to $\mathrm{Sn}$, which has the strongest isotope effect in nature where $T_{c}$ varies only by $4 \%$. For each family, we measured the Néel temperature $T_{N}$ and the anisotropies of the magnetic interactions. This allows us to obtain the Heisenberg coupling $J$. In addition, we determine the spin-glass temperature $T_{g}$ of underdoped samples. $J, T_{g}$, and $T_{c}$ allow us to generate a unified phase diagram for magnetism and superconductivity from no doping to overdoping. We combine this result with a previous determination of the superconducting carrier density $n_{s}{ }^{3}$ and demonstrate experimentally a magnetic analog of the isotope effect.

CLBLCO is a high-temperature superconductor (HTSC) system that belongs to the $\mathrm{YBa}_{2} \mathrm{Cu}_{3} \mathrm{O}_{7}$ (YBCO) group. Each value of $x$ in the range of $0.1 \leqslant x \leqslant 0.4$ (Ref. 4) is a family in the system. All families are tetragonal. The difference in the unit-cell parameters $a$ and $c / 3$ between the two extreme families $(x=0.1$ and 0.4$)$ is $1 \% .^{4}$ Therefore, variations in $T_{c}^{\max }$ due to variation in ionic radios are not relevent since they are expected to be on the order of $1 \mathrm{~K}$, and with opposite sign to observation. ${ }^{5}$ Moreover, the level of disorder as detected by Ca NMR (Ref. 6) and Cu NQR (Ref. 7) is identical for the different families. The range of accessible $y$ 's in CLBLCO is so large that samples from the heavily underdoped antiferromagnetic (AFM) parent compounds, to the nonsuperconducting extreme overdoped, are obtainable. When varying $x$, nearly concentric domes of $T_{c}$ versus oxygen doping $y$ are formed, with the maximum value of $T_{c}$ varying from $58 \mathrm{~K}$ at $x=0.1$ to $80 \mathrm{~K}$ at $x=0.4,{ }^{8}$ as demonstrated by the open symbols in Fig. 1. The magnetic properties of these compounds are determined using the zero-field muon spin-relaxation ( $\mu \mathrm{SR})$ technique.

Figure 2 shows typical muon polarization $P(t)$ curves, at different temperatures, for three samples from the $x=0.1$ family. At high temperatures the polarization curves from all samples are typical of magnetic fields emanating from nuclear magnetic moments. In this case the time dependence of the polarization exhibits, as expected, a Gaussian decay. As the temperature is lowered the sample enters a magnetic frozen phase and the polarization relaxes much more rapidly. While the transition from paramagnetic to the frozen state looks identical for all samples, the behavior at very low $T$ is different and indicates the nature of the ground state. Figure $2(a)$ is an example of an antiferromagnetic ground state. When the temperature decreases, long-range magnetic order is established at $\sim 377 \mathrm{~K}$ reflected by spontaneous oscillations of the muon polarization. Figure 2(c) is an example for a spin-glass $(\mathrm{SG})$ transition at $\sim 17 \mathrm{~K}$. In this case the ground state consists of magnetic islands with randomly frozen electronic moments, ${ }^{9}$ and consequently, the polarization shows only rapid relaxation. When the transition is to a Néel or spin-glass state, the critical temperatures are named $T_{N}$ and $T_{g}$, respectively. Figure 2(b) presents an intermediate case where the sample appears to have two transitions. The first

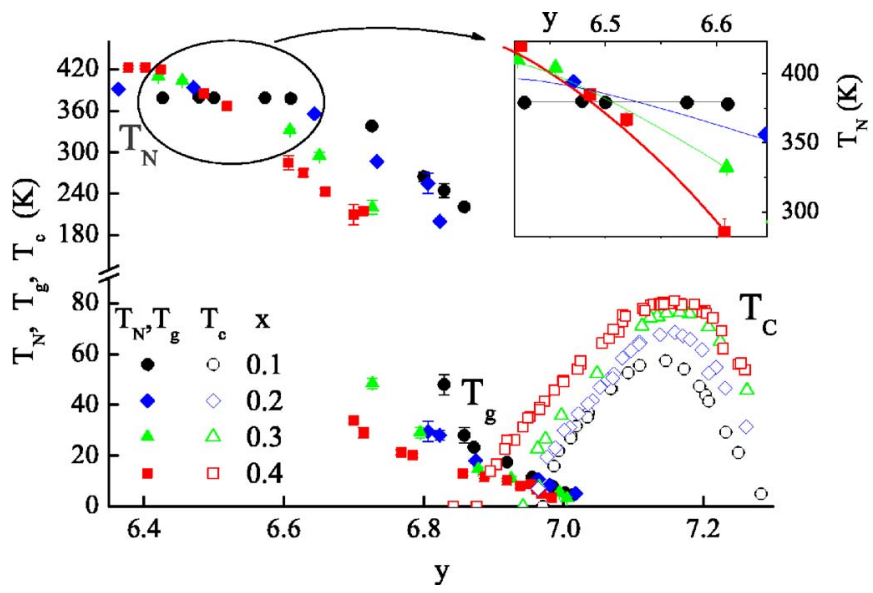

FIG. 1. (Color online) Phase diagram for the $\left(\mathrm{Ca}_{x} \mathrm{La}_{1-x}\right)\left(\mathrm{Ba}_{1.75-x} \mathrm{La}_{0.25+x}\right) \mathrm{Cu}_{3} \mathrm{O}_{y}$ system. 


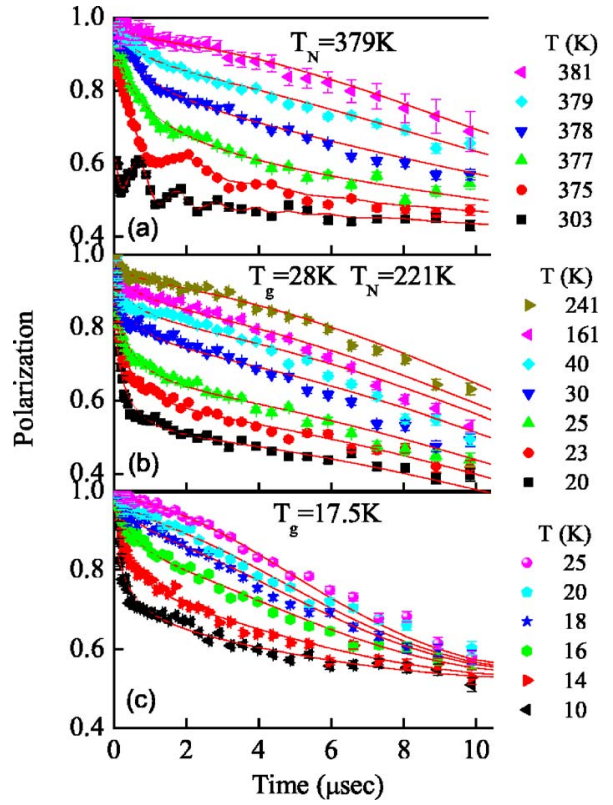

FIG. 2. (Color online) Time evolution of the muon polarization for $\left(\mathrm{Ca}_{x} \mathrm{La}_{1-x}\right)\left(\mathrm{Ba}_{1.75-x} \mathrm{La}_{0.25+x}\right) \mathrm{Cu}_{3} \mathrm{O}_{y}$ from the $x=0.1$ family close to the magnetic critical temperature. (a) A sample with an antiferromagnetic transition. (b) A sample with both an antiferromagnetic and a spin-glass transition. (c) A sample with a spin-glass transition. The solid lines are a fit to Eq. (1).

one starts below $240 \mathrm{~K}$, where the fast decay in the polarization appears. Between 160 and $40 \mathrm{~K}$ there is hardly any change in the polarization decay, and at $30 \mathrm{~K}$ there is another transition manifested in a faster decaying polarization. This behavior was observed in all the samples on the border between antiferromagnet and spin glass in the phase diagram.

In order to determine the magnetic critical-transition temperatures, the data were fitted to a sum of two functions: a Gaussian, and a rapidly relaxing function that describes the magnetic field due to frozen electronic moments, namely,

$$
\begin{aligned}
P(t)= & A_{n} \exp \left(\frac{-\Delta^{2} t^{2}}{2}\right)+A_{m}\left\{a \exp \left(-\sqrt{\lambda_{1} t}\right)\right. \\
& \left.+(1-a) \exp \left(-\sqrt{\lambda_{2}} t\right) \cos (\omega t)\right\} .
\end{aligned}
$$

In this function $A_{n}$ and $A_{m}$ represent the amplitudes (i.e., the volume fraction) of the nuclear and magnetic parts, respectively, and $\lambda_{1}, \lambda_{2}$ are the relaxation rates of the magnetic part. In the fit $\Delta$ is determined from high temperatures, and the sum $A_{m}+A_{n}=1$ is constant at all temperatures. The solid lines in Fig. 2 are the fits of Eq. (1) to the data. Figure 3 shows $A_{m}$ as a function of temperature for the three samples in Fig. 2. Above the transition, where only nuclear moments contribute, $A_{m}$ is close to zero. As the temperature decreases, the frozen magnetic part increases and so does $A_{m}$, at the expense of $A_{n}$. For the pure AFM and SG phases, the transition temperature was determined as the temperature at which $A_{m}$ is half of the saturation value. For the samples with two transitions, two temperatures were determined using the same principle. The coexistence of Néel and spin-glass states indicates that the transition from one kind of ground state to

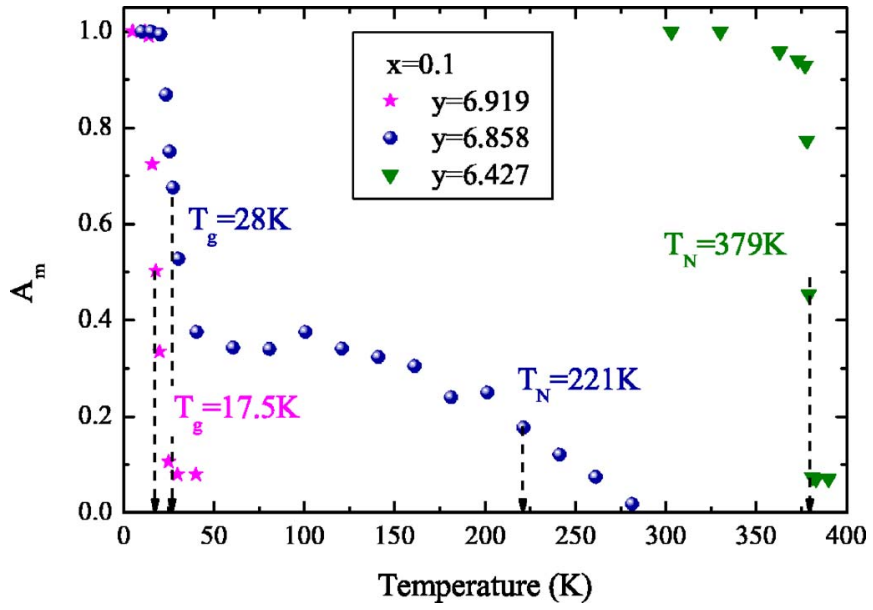

FIG. 3. (Color online) The magnetic volume fraction extracted from the muon depolarization as a function of temperature for the three samples shown in Fig. 2.

the other is a first-order quantum phase transition.

The full phase diagram of the CLBLCO compound, obtained from the measurements described above, including the $T_{g}$ measurements in the superconducting state from Ref. 9, is presented in Fig. 1. From this diagram it is clear that, at high doping levels, CLBLCO is a pure superconductor. As the oxygen doping decreases considerably from optimum, these compounds can be in a mixed state of magnetic islands, which freeze at $T_{g}$, and superconductivity in between. Upon further decrease in doping, the system has a pure spin-glass ground state. Even further underdoping towards the parent compounds leads to abrupt replacement of the glassy state with a long-range antiferromagnetic order with $T_{N} \sim 400 \mathrm{~K}$, which seems to saturate. The inset of Fig. 1 shows that $y$ $\sim 6.5$ is a crossing point, and at higher doping, families with high $x$ values have lower $T_{N}$ and $T_{g}$. In contrast, at doping levels below $y=6.5$, families with higher $x$ values (and higher $T_{c}^{\max }$ ) have the higher $T_{N}$. This is the first indication of correlation between $T_{c}^{\max }$ and magnetic interactions.

In order to untangle the phase diagram, we use the scaling relation introduced in Ref. 9 that caused both $T_{c}$ and $T_{g}$ data of many cuprate families to collapse into a single curve. ${ }^{10}$ This means plotting, for each family, $T_{c}$ divided by $T_{c}^{\max }$ of the family, as a function of $\Delta P$ where $\Delta P=K(x) \Delta y, \Delta y=y$ $-y_{0}$ is the chemical doping measured from optimum $y_{0}$, and $K(x)$ is the scaling parameter determined for each family so that all the superconducting domes collapse onto a single curve. ${ }^{9}$ We use $K=0.77,0.67,0.54,0.47$, and $y_{0}=7.135$, $7.15,7.155,7.15$ for the $x=0.1-0.4$, respectively. The results are presented in the upper inset of Fig. 4. We find that, with the exception of the $x=0.1$ family in the AFM region, the scaling relation holds perfectly well for the entire phase diagram.

Nevertheless, we would like to check whether the scaling relation can be extended to the $x=0.1$ family. We suspected that in $x=0.1$ anisotropies might be different from the other families. It is well established that a pure two-dimensional (2D) AFM orders magnetically only at $T=0$, and that $T_{N}$ for three-dimensional (3D) AFM is finite. Intermediate cases are 


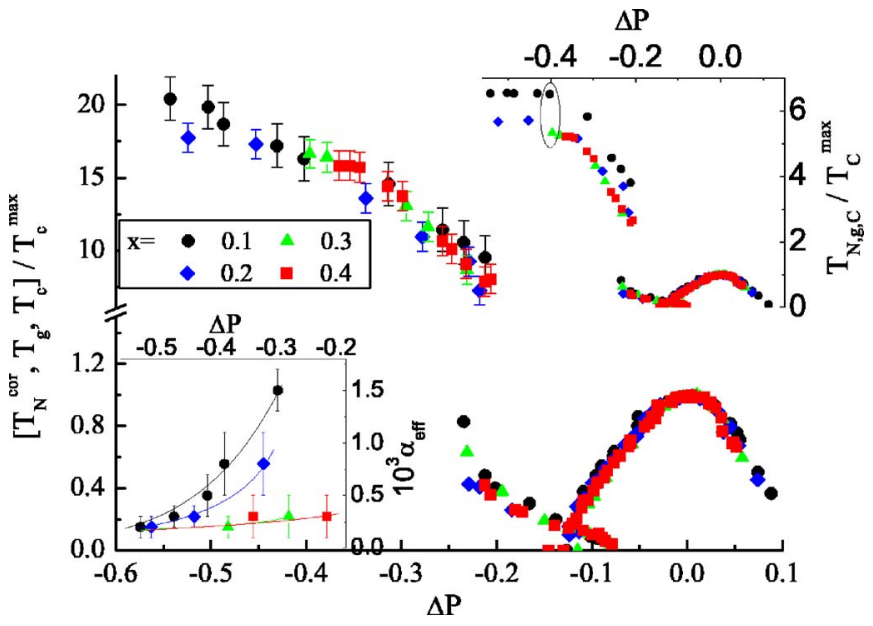

FIG. 4. (Color online) Upper inset: the phase diagram after the scaling described in the text. Lower inset: The effective anisotropies as a function of $\Delta P$ for the different families (see the text for details). The solid lines are guides to the eye. Main figure: phase diagram after both scaling and extraction of the contributions from anisotropies as described in the text. $T_{N}^{c o r}=J$ for the parent compounds.

described by anisotropic interactions where $J$ and $\alpha J$ are the in- and out-of-plane coupling, respectively. For small $\alpha$ the reduction of the magnetic-order parameter $M$ with increasing $T$ is fast so that at $\alpha=0$ the 2D limit is recovered. A plot of the normalized order parameter $\sigma=M / M_{0}$, where $M_{0}$ is the order parameter at $T \rightarrow 0$, versus $T / T_{N}$ should connect $(1,0)$ to $(0,1)$ (see Fig. 5) in a way that is determined only by $\alpha$. At the same time $J \propto T_{N} \ln (\alpha)$ (up to $\ln$ of $\ln$ of $\alpha$ corrections) ${ }^{11}$ so that knowledge of $\alpha$ would lead to $J$.

To test our hypothesis we measure $P(t)$ with high timing resolution at $T \rightarrow 0$, and extract $\sigma$. A typical time-dependent

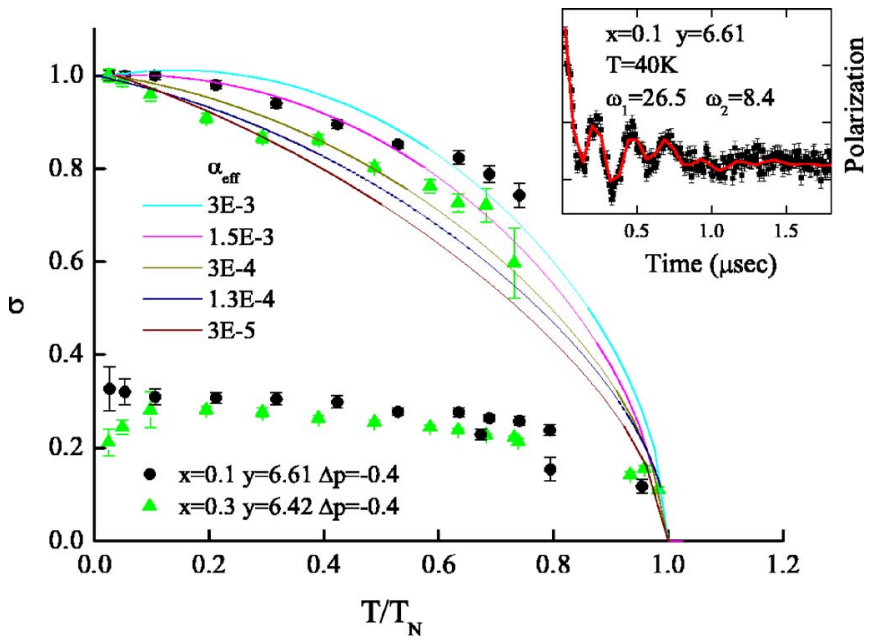

FIG. 5. (Color online) The normalized staggered magnetization as a function of the normalized temperature. The symbols are the experimental results, taken by measuring the oscillation frequency of the polarization curves. The solid lines are the theoretical curves plotted according to Eqs. (4)-(7). Inset: an example of muon polarization for a sample in the Néel state at $T \rightarrow 0$; the solid line is a fit to Eq. (2). polarization is depicted in the inset of Fig. 5. The best fit of the polarization is achieved with the function

$$
P(t)=\sum_{i=1}^{3} A_{i} \exp \left(-\lambda_{i} t\right) \cos \left(\omega_{i} t\right)
$$

with $\omega_{3}=0$, which is shown in the inset by the solid line. The reason for multiple frequencies is that the muons stop at different sites in the unit cell. Since the muon oscillation frequency is proportional to the local magnetic field it experiences, $\omega(T) / \omega_{0}$ is equivalent to $\sigma$. The order parameter extracted from the high frequency, around a few tens of $\mathrm{MHz}$ $\left(\omega_{0} \sim 27 \mathrm{MHz}\right.$ in our case) is known to agree with neutronscattering determination of $\sigma .{ }^{12}$ The lower frequency is believed to emerge from metastable muon sites and is not used for further analysis.

In Fig. 5 we present $\sigma$ for the two different CLBLCO samples with $x=0.1$ and 0.3 having the same $\Delta P$ that is marked in the inset of Fig. 4. The data sets starting at $\sigma=1$ are from the high frequency and will be used for comparison with theory. Clearly, the reduction of the magnetization with increasing temperatures is not the same for these two samples, and therefore their anisotropies are different. Since $\sigma$ is less sensitive to increasing $T$ in the $x=0.1$ family than in the $x=0.3$ family, the $\alpha$ of $x=0.1$ must be larger. Consequently, $T_{N}$ of the $x=0.1$ turns out to be too high due to $\alpha$ and not $J$. This could explain the deviation from scaling of the $x=0.1$ family.

To account for the anisotropies quantitatively we assume that CLBLCO could be considered as a 2D magnet with weak anisotropies since the chain layers are partially full with oxygen even for the parent compounds. Therefore we use the Hamiltonian

$$
H=J\left(\sum_{i, \delta_{\|}} \mathbf{S}_{i} \cdot \mathbf{S}_{i+\delta_{\|}}+\alpha_{x y} \sum_{i, \delta_{\|}} S_{i}^{z} S_{i+\delta_{\|}}^{z}+\alpha_{\perp} \sum_{i, \delta_{\perp}} \mathbf{S}_{i} \cdot \mathbf{S}_{i+\delta_{\perp}}\right),
$$

where $\delta_{\|}$and $\delta_{\perp}$ are the in- and out-of-plane neighbor spacings, respectively. We apply the self-consistent Schwingerboson mean-field (SBMF) theory ${ }^{13}$ to calculate $\sigma\left(\alpha_{\text {eff }}, t\right)$ where $t=T / J, \alpha_{e f f}=z_{x y} \alpha_{x y}+z_{\perp} \alpha_{\perp}$, and the $z$ 's are the number of neighbors. The calculation is done by solving simultaneously for every $\alpha_{e f f}$ and $t$ two equations: a self-consistency equation

$$
h=2 \alpha_{e f f}[1-2 K(\Delta, h, t)],
$$

and a constraint equation ensuring one Schwinger-boson per site,

$$
K(\Delta, h, t)+K(\Delta, 0, t)=1 .
$$

In these equations

$$
K(\Delta, h, t)=2.32 \int_{0}^{1} \frac{(1+\Delta+h)}{\omega(\Delta, h, \gamma)}[n(\omega(\Delta, h, \gamma), t)+1 / 2] \rho(\gamma) d \gamma
$$

the density of states is given by 


$$
\rho(\gamma)=\frac{2}{\pi^{2}} \int_{0}^{1}\left[\left(1-t^{2}\right)\left(1-t^{2}+\gamma^{2} t^{2}\right)\right]^{-1 / 2} d t,
$$

or its approximation, ${ }^{14,15} \quad \omega(\Delta, h, \gamma)=2.32\left[(1+\Delta+h)^{2}\right.$ $\left.-\gamma^{2}\right]^{1 / 2}$, and $n(\omega, t)=[\exp (\omega / t)-1]^{-1}$. Finally, $\sigma\left(\alpha_{e f f}, t\right)$ $=h\left(\alpha_{e f f}, t\right) / h\left(\alpha_{e f f}, 0\right) . t_{N}$ and $T_{N}$ are defined by $\sigma\left(\alpha_{e f f}, t_{N}\right)$ $=0$ and $T_{N}=J t_{N}$. The theoretical order parameter for several $\alpha_{e f f}$ as a function of $T / T_{N}$ is also presented in Fig. 5 by the solid lines.

When comparing theory and experiment we focus on the low-temperature data, up to $200 \mathrm{~K}$, where the theory is most accurate. We determine the best $\alpha_{\text {eff }}$ and present them in the lower inset of Fig. 4. The solid lines are guides to the eye. Using the corresponding $t_{N}\left(\alpha_{e f f}\right)$ we obtain the corrected $T_{N}$ defined as

$$
T_{N}^{c o r} \equiv T_{N} / t_{N}\left(\alpha_{e f f}\right),
$$

where $T_{N}^{c o r}=J$ for the parent compounds. At $\Delta P=-0.4$, where $T_{N}$ of all samples is saturated and they can be compared, we find $J=950(60), 1130(70), 1260(80)$, and $1330(80) \mathrm{K}$ for $x$ $=0.1,0.2,0.3$, and 0.4 , respectively.

This variation of $J$ is probably due to an increasing buckling angle and decreasing lattice constants with increasing $x$. Neutron diffraction shows that the buckling angle of the optimally doped samples changes by 1.3 degrees from $x=0.1$ to $x=0.4$. Similarly, the lattice constant $a$ changes by $0.03 \AA .{ }^{17,18}$ According to Ref. 19, where $J$ is plotted as a function of the $\mathrm{Cu}-\mathrm{O}-\mathrm{Cu}$ buckling angle and bond length for a variety of materials, this king of changes can produce a $30 \%$ variation in $J$.

Determining $t_{N}$ experimentally for the AFM compounds allows us to present a modified phase diagram using $T_{N}^{c o r}$. The modified phase diagram showing $T_{N}^{c o r}, T_{g}$, and $T_{c}$ nor- malized by $T_{c}^{\max }$ versus $\Delta P$ is presented in Fig. 4 . Note, $T_{g}$ is not corrected since it is determined by interactions between islands, and anisotropies are not expected to play an important role in this case. The large error bars for $T_{N}^{c o r}$ are due to the pure determination of $t_{N}\left(\alpha_{e f f}\right)$. Nevertheless, the scaling now works for all four families, with a $T_{c}^{\max }$ variation of $28 \%$, in most of the AFM region as well. This demonstrates that an energy scale $J_{f}$, unique for each family but varying between families, controls both $T_{N}, T_{g}$, and $T_{c}$.

In a previous work it was found that CLBLCO obeys the Uemura relation $T_{c} \propto n_{s},{ }^{16}$ where $n_{s}$ is the superconducting carrier density, in both under- and overdoped regions. ${ }^{3} \mathrm{Com}-$ bining this fact with our present finding that a magnetic energy scale controls $T_{c}^{\max }$, suggests the formula

$$
T_{c}=c J_{f} n_{s}(\Delta P),
$$

where the constant $c=0.7(1)$ (using the definition $n_{s}(0)$ $=0.08$ carrier per $\mathrm{Cu}$ ), $\Delta P=0$ at optimal doping for all families, and $n_{s}$ is family independent.

Equation (9) is a magnetic equivalent of the isotope effect $T_{c}=c M^{-1 / 2}$. We demonstrate this magnetic effect for $15 \%$ variation of $T_{c}^{\text {max }}$ without any theory. This is far greater than the $T_{c}$ variation due to the strongest isotope effect in metallic superconductors. Using the SBMF theory we extend the effect further to $28 \%$ of $T_{c}^{\max }$. Therefore, our data are a strong support to the belief that magnons are responsible for producing pairing in the cuprates.

We would like to thank the ISIS pulsed muon facility at Rutherford Appleton Laboratory, UK and the $\mathrm{S} \mu \mathrm{S}$ facility at Paul Scherrer Institute, Switzerland. This work was funded by the Israeli Science Foundation and the Posnansky Research Fund in high-temperature superconductivity.
${ }^{1}$ E. Maxwell and O. S. Lutes, Phys. Rev. 95, 333 (1954).

${ }^{2}$ A. V. Chubukov, D. Pines, and B. P. Stojković, J. Phys.: Condens. Matter 8, 10017 (1996); E. Altman and A. Auerbach, Phys. Rev. B 65, 104508 (2002); D. Muñoz, I. de P. R. Moreira, and F. Illas, ibid. 65, 224521 (2002); P. W. Anderson, P. A. Lee, M. Randeria, T. M. Rice, N. Trivedi, and F. C. Zhang, J. Phys.: Condens. Matter 16, R755 (2004); E. Demler, W. Hanke, and S.-C. Zhang, Rev. Mod. Phys. 76, 909 (2004); S. A. Kivelson and E. Fradkin, cond-mat/0507459 (unpublished).

${ }^{3}$ A. Keren, A. Kanigel, J. S. Lord, and A. Amato, Solid State Commun. 126, 39 (2003).

${ }^{4}$ D. Goldschmidt et al., Phys. Rev. B 48, 532 (1993).

${ }^{5}$ X.-J. Chen and H. Su, Phys. Rev. B 71, 094512 (2005).

${ }^{6}$ Sebastien Marchand, Ph.D. thesis, Université Paris 6 (2005).

${ }^{7}$ A. Keren, Amit Kanigel, and Galina Bazalitsky, Phys. Rev. B 74, 172506 (2006).

${ }^{8}$ A. Knizhnik et al., Physica C 321, 199 (1999).
${ }^{9}$ A. Kanigel, A. Keren, Y. Eckstein, A. Knizhnik, J. S. Lord, and A. Amato, Phys. Rev. Lett. 88, 137003 (2002).

${ }^{10}$ A. Keren and A. Kanigel, Phys. Rev. B 68, 012507 (2003).

${ }^{11}$ B. Keimer et al., Phys. Rev. B 45, 7430 (1992).

${ }^{12}$ A. Keren et al., Phys. Rev. B 48, 12926 (1993).

${ }^{13}$ D. P. Arovas and A. Auerbach, Phys. Rev. B 38, 316 (1998).

${ }^{14} \mathrm{~A}$. Auerbach, Interacting Electrons and Quantum Magnetism (Springer-Verlag, New York, 1994).

${ }^{15}$ M. Abramovitz and I. A. Stegun, Handbook of Mathematical Functions (Dover Publications, Inc., New York, 1970).

${ }^{16}$ Y. J. Uemura et al., Phys. Rev. Lett. 62, 2317 (1989).

${ }^{17}$ R. Khasanov, D. G. Eshchenko, H. Luetkens, E. Morenzoni, T. Prokscha, A. Suter, N. Garifianov, M. Mali, J. Roos, K. Conder, and H. Keller, Phys. Rev. Lett. 92, 057602 (2004).

${ }^{18}$ O. Chmaissem et al., Nature (London) 397, 45 (1999).

${ }^{19}$ G. V. R. Chandramouli, T. K. Kundu, and P. T. Manoharan, Aust. J. Chem. 56, 1239 (2003). 